XXVII International Conference on Photonic, Electronic and Collisions

XXVII ICPEAC

BELFAST 2011

Home

Dates/Announcements

Scope and Programme Awards at ICPEAC

Conference venue

Accommodation

Registration

Travel

Abstracts

- Submission

- Search

- Posters

Proceedings

Social programme

Satellite meetings

Exhibitors/Sponsors

Local research

About ICPEAC

Notice board

Contacts
27 July - 2 August 2011, Belfast, Northern Ireland, UK

\section{Search Results}

2 abstracts have been found.

New Search

$\underline{1}$

Electron and Positron Scattering from Pyrimidine

P. D. Palihawadana , J. R. Machacek , C. Makochekanwa , J. P. Sullivan , M. J. Brur McKoy , G. Garcia , F. Blanco, S. J. Buckman

Modelling low energy electron and positrons tracks for biomedical applicat

A.G. Sanz, M.C. Fuss , A.M. Roldan, J.C. Oller, F. Blanco, P.Limao-Vieira, M.J.Br G.Garcia

1 


\title{
Modelling low energy electron and positron tracks for biomedical applications
}

\author{
A. G. Sanz*, M. C. Fuss*, A. M. Roldán ${ }^{\dagger}$, J. C. Oller ${ }^{\dagger}$, F. Blanco ${ }^{\#}$, P. Limão-Vieira ${ }^{\ddagger}$, \\ M. J. Brunger ${ }^{\S}$, S. J. Buckman ${ }^{\mathfrak{1}}$ and G. García* ${ }^{1}$ \\ *Instituto de Física Fundamental, CSIC, Serrano 113-bis, 28006 Madrid, Spain \\ ${ }^{\dagger}$ CIEMAT, Avenida Complutense 22, 28040 Madrid, Spain. \\ ${ }^{\#}$ Departamento de Física Atómica, Molecular y Nuclear, Universidad Complutense de Madrid, 28040 Madrid. \\ "Departamento de Física, CEFITEC, FCT-Universidade Nova de Lisboa, 2829-516 Caparica, Portugal. \\ ${ }^{\S}$ ARC Centre for Antimatter-Matter Studies, School of Chemical and Physical Sciences, Flinders University, G.P.O. \\ Box 2100, Adelaide, South Australia 5001, Australia. \\ ${ }^{£}$ ARC Centre for Antimatter-Matter Studies, Research School of Physics and Engineering, Australian National \\ University, Canberra 0200 Australia.
}

\begin{abstract}
Synopsis In order to incorporate the effect of low energy electrons and positron in radiation damage models, the simulation method proposed here is based on experimental and theoretical cross section data and energy loss spectra we have previously derived. After a summary of the main techniques used to obtain reliable input data, the basis of a Low Energy Particle Track Simulation (LEPTS) procedure is established. Single electron and positron tracks in liquid water are presented and the possibility of using these results to develop tools for nanodosimetry is discussed.
\end{abstract}

Biomedical uses of radiation are demanding an increased level of detail in order to describe the interaction processes initiating radiation damage. In particular, reduced volume irradiation techniques such as brachytherapy [1], in which radiation emitters are placed close to the target, not only require traditional dosimetric methods to prescribe the treatment but also for the evaluation of possible side effects in surrounding sensitive areas.

The important role of low energy, sub-ionising, secondary electrons to induce damage in biomolecular systems has been widely studied in the last decade $[2,3]$. According to these studies, realistic radiation interaction models should include low energy electron interactions up to their final thermalisation in the medium. In addition, the interest in developing similar models for positrons is rapidly increasing as their use in Positron Emission Tomography (PET) is requiring a better understanding of the physical processes underlying this technique.

Recently, this need of describing radiation effects at the molecular level has led to the development of the concept of nanodosimetry, namely a procedure to quantify radiation damage in nano-volumes. Absorbed dose is not obviously a proper parameter to describe effects at the nanoscale, rather a detailed description of the interaction processes occurring in a nano-size target and their implications in terms of radiation damage (number of dissociative events, type of radicals generated, etc...) are what is really required. As this nano-region of interest could be relatively far (tens of microns or even millimetres) from the original track of the incident high energy primary particles, an accurate description of single tracks of secondary particles will be essential for that purpose.

These reasons motivated the present study in which we describe a new low energy particle track simulation (LEPTS) code, especially designed to provide interaction details at the nano-scale. The simulation procedure is based on a step by step Monte Carlo code, which uses as input parameters the experimental and theoretical electron and positron scattering cross sections and energy loss distribution functions we have previously obtained. Information about the experimental and theoretical methods used for this purpose, as well as input data we used for the electron and positron interactions in water, will be presented. We also provide details about the simulation procedure. Finally, single electron and positron tracks in water will be shown to provide examples of output data capable to quantify radiation damage in nano-volumes.

\section{References}

[1] M Fuss et al 2009 Rev. Esp. Fis 2340

[2] B Boudaïffa et al 2000 Science 2871658

[3] M A Huels et al 2003 JACS 1254467

${ }^{1}$ E-mail: g.garcia@iff.csic.es 